\title{
Ruptured Internal Iliac Artery Aneurysm: Staged Emergency Endovascular Treatment in the Interventional Radiology Suite
}

\author{
Bas-Jeroen van Kelckhoven · Boy M. A. Bruijninckx • \\ Bob Knippenberg $\cdot$ Hans van Overhagen
}

Published online: 10 April 2007

(C) Springer Science+Business Media, LLC 2007

\begin{abstract}
Ruptured aneurysms of the internal iliac artery (IIA) are rare and challenging to treat surgically. Due to their anatomic location they are difficult to operate on and perioperative morbidity is high. An endovascular approach can be helpful. We recently treated a patient with a ruptured IIA aneurysm in the interventional radiology suite with embolization of the side-branch of the IIA and placement of a covered stent in the ipsilateral common and external iliac arteries. A suitable stent-graft was not available initially and had to be brought in from elsewhere. An angioplasty balloon was temporarily placed across the ostium of the IIA to obtain hemostasis. Two hours later, the procedure was finished by placing the stent-graft.
\end{abstract}

The purpose of treatment of an aneurysm is to exclude it from the circulation and to restore distal vascularization. In patients with a ruptured aneurysm, emergency treatment is warranted. Two methods of treatment are currently available: open surgery and endovascular aneurysm repair (EVAR). Due to their location internal iliac artery (IIA) aneurysms are not directly accessible for stent-graft placement. Thus, endovascular treatment consists of excluding the vessel from the circulation by coiling the side-branches of the IIA and placing a covered stent in the ipsilateral common iliac artery (CIA) and external iliac artery (EIA) covering the origin of the IIA. In this report we present a patient with acute rupture of an IIA aneurysm

B.-J. van Kelckhoven $(\bowtie) \cdot$ H. van Overhagen Department of Radiology, HagaZiekenhuis, Location Leyenburg, Leyweg 275, 2545 CH Den Haag, The Netherlands e-mail: bjvankelckhoven@ @otmail.com

B. M. A. Bruijninckx · B. Knippenberg

Department of Surgery, HagaZiekenhuis, Location Leyenburg, Leyweg 275, 2545 CH Den Haag, The Netherlands that was successfully treated with endovascular techniques in the interventional radiology suite.

\section{Case Report}

A 72-year-old obese man was admitted to our hospital with a 1 week history of abdominal pain, located mainly in the right lower quadrant. On physical examination there was abdominal tenderness, mainly on the right side. Blood pressure was $145 / 92 \mathrm{mmHg}$; heart rate was 120 beats/min. Blood analysis showed some elevated liver enzymes and a hemoglobin level of $9.0 \mathrm{mmol} / \mathrm{l}$. During investigation of the patient, his condition deteriorated, his heart rate rose to above 120 beats/min, and systolic blood pressure fell below $100 \mathrm{mmHg}$.

Abdominal sonography showed free intra-abdominal fluid and a large aneurysm in the right lower abdomen. At sonographic-guided puncture blood was aspirated. Subsequently CT-angiography (CTA) was performed which showed an aneurysm of the right IIA with a size of $7.5 \mathrm{~cm}$ and a discontinuous irregular ring of calcifications in the vessel wall (Fig. 1). Perivascular stranding and intraabdominal fluid indicated rupture. Only one of the descending branches of the right IIA seemed patent. In addition there was an aneurysm of the left CIA with a diameter of $3.0 \mathrm{~cm}$, which was not ruptured.

Two treatment options were considered: open surgery or endovascular treatment. The development of hypovolemic shock and the patient's morbid obesity, combined with the known difficulties of treating a large IIA aneurysm by open surgery, made us decide to attempt endovascular treatment $[1,2]$. Because of continuing leakage of the aneurysm hemostasis had to be obtained first. The patient was taken to the interventional radiology suite under continuous 
Fig. 1 a, b. Axial and coronal reconstructions of CTangiography. a A large aneurysm of the internal iliac artery (white arrows) with surrounding blood (black arrow) is seen on the right side. b On the left side a smaller aneurysm of the common iliac artery is seen without signs of rupture (white arrowhead)
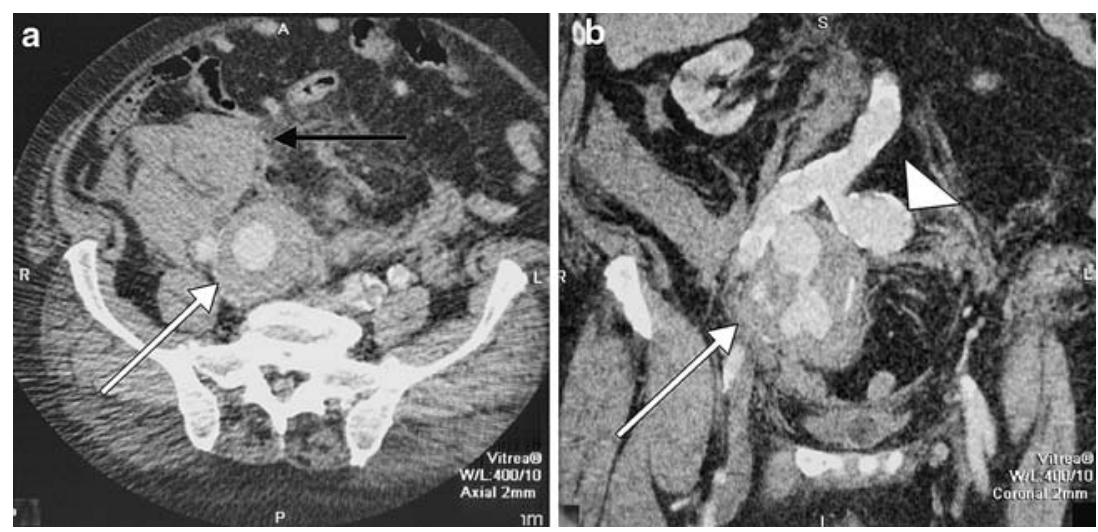

monitoring of heart rate and blood pressure. After local anesthesia a $6 \mathrm{Fr}$ introducer sheath was placed in the right common femoral artery, using the Seldinger technique. Digital subtraction angiography of the distal aorta and right iliac artery confirmed the CTA findings. A right-sided IIA aneurysm with only one side-branch was seen. In addition there was a perivascular blush of contrast medium due to contrast extravasation adjacent to the aneurysm, indicating active bleeding (Fig. 2a). The IIA and its side-branch were selectively catheterized with a 5 Fr Cobra 1 catheter (Cordis, Roden, The Netherlands). Four coils $-2 \times 9 \mathrm{~mm}$ (Boston Scientific, Cork, Ireland), $1 \times 12 \mathrm{~mm}$, and $1 \times 15$ mm (Cook, Bjaeverskov, Denmark)—were placed selectively in the side-branch of the IIA (Fig. 2b). Additionally, Gelfoam particles were injected to enhance hemostasis. A covered stent-graft had to be placed, across the origin of the IIA. However, due to the relatively large sizes (diameters $16-20 \mathrm{~mm}$ ) of the ipsilateral common and external iliac arteries, no suitable stent-graft was available in our hospital at that time and one had to be brought in from a company warehouse elsewhere. To obtain temporary hemostasis a $15 \mathrm{~mm} \times 40 \mathrm{~mm}$ angioplasty balloon (Cordis, Roden, The Netherlands) was placed in the right CIA and EIA, across the ostium of the IIA. At this moment the patient was out of direct danger because the aneurysm was considered to be excluded from the circulation.

During the time it took to deliver the stent-graft, the patient was stabilized in the intensive care unit (ICU). When the stent-graft arrived we continued the procedure. The angioplasty balloon was removed and the $6 \mathrm{Fr}$ introducer sheath was exchanged for an $18 \mathrm{Fr}$ introducer sheath using a stiff 0.035-inch Lunderquist guidewire (both: Cook, Bjaeverskov, Denmark). A $135 \mathrm{~mm}$ iliac leg of an Excluder endovascular graft (Gore, Flagstaff, AZ, USA) with a proximal diameter of $16 \mathrm{~mm}$ and distal diameter of $20 \mathrm{~mm}$ was positioned in place and released. We subsequently dilated the stent-graft with a $15 \mathrm{~mm}$ angioplasty balloon in the EIA and with a $20 \mathrm{~mm}$ balloon in the proximal CIA (both: Cordis, Roden, The Netherlands). After dilatation, angiography showed a patent stent-graft without endoleak. The blush of contrast medium at the site of the rupture was no longer observed (Fig. 3). The $18 \mathrm{Fr}$ sheath was left in place, as the access site was considered too large to close with manual compression. The patient was transferred to the ICU and remained stable overnight. No blood loss was reported, the hemoglobin level remained stable, and the next day the patient was transferred to the surgical ward. The 18 Fr sheath was removed surgically under local anesthesia. Repeat CTA 1 week after the procedure showed a patent stent-graft without signs of endoleak (Fig. 4). At present, at 2 months follow-up, the patient is clinically doing fine.

\section{Discussion}

Isolated aneurysms of the IIA are rare $(0.3-0.5 \%$ of all aorto-iliac aneurysms) and are most often observed in the
Fig. 2 a Angiography of the common iliac artery on the right side. The IIA is

aneurysmatically enlarged (white arrow) and a contrast blush indicating active bleeding is seen outside the vessel lumen (black arrows). There is only one vessel branching from the IIA. b Four coils are placed in the only vessel originating from the right IIA (arrowhead)
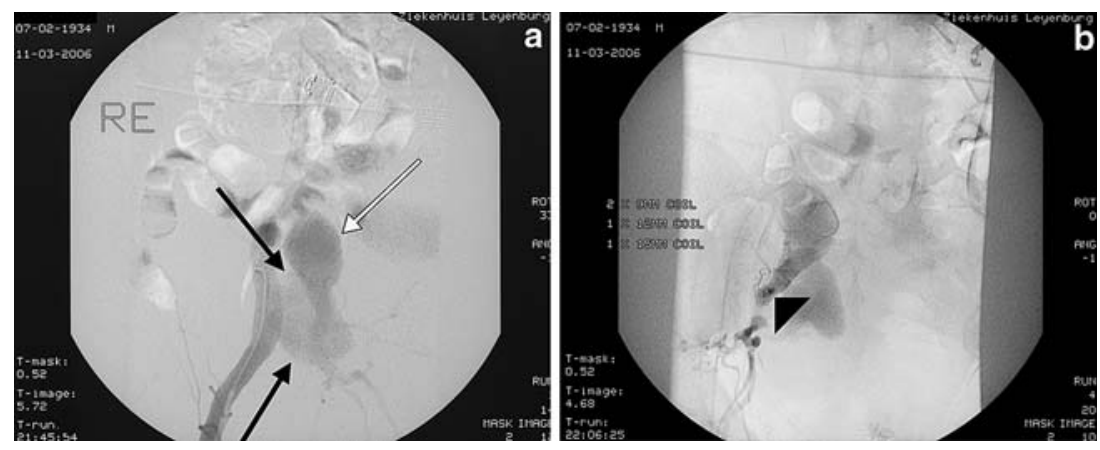


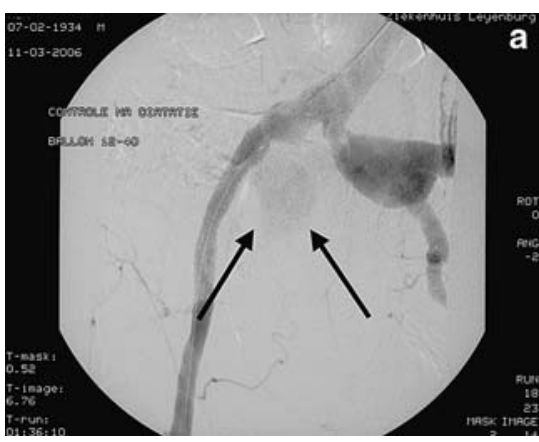

Fig. 3 a, b. Angiography after coiling the right IIA and placing a stent-graft in the common iliac and external iliac arteries. a After the first dilatation with the angioplasty balloon, a faint blush of contrast

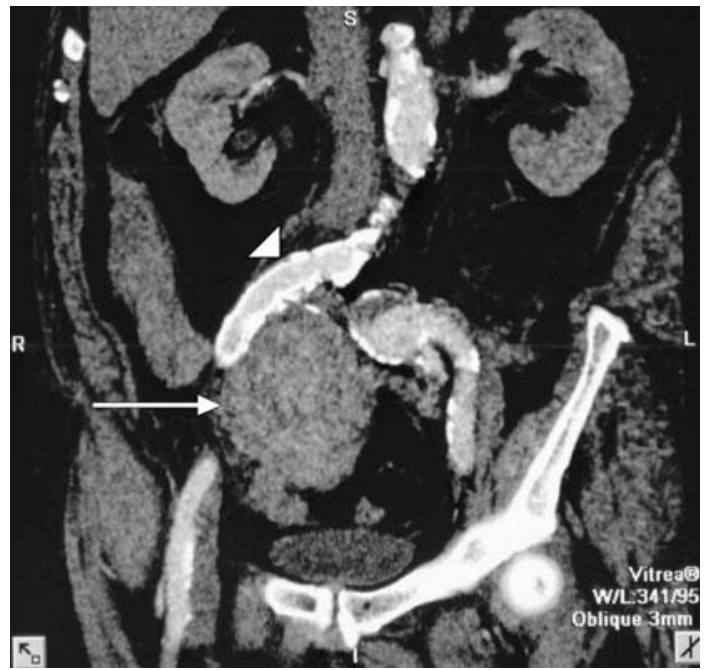

Fig. 4 Repeat CT-angiography, 1 week after the procedure. Coronal reconstruction shows a patent stent-graft (white arrowhead) and no signs of endoleak. No contrast enhancement of the aneurysm (white arrow) is seen

male population [1]. IIA aneurysms frequently are asymptomatic and found during examinations performed for other purposes. When initially detected the aneurysms are usually relatively large, with a median size of $7.7 \mathrm{~cm}$ [1]. Symptoms at presentation may consist of rupture with pain and hemodynamic instability or compression of either the genitourinary system or the lumbosacral nerves. The incidence of rupture is reported to be as high as $33 \%$ at initial presentation with a mortality rate at open surgical repair of $33-50 \%[1,2]$. In addition morbidity is high because of peroperative blood loss and possible buttock ischemia [1]. Aneurysms close to the origin of the IIA are especially difficult to operate on. Endovascular treatment is certainly more attractive in these cases. Advantages of endovascular treatment are fast hemostasis and limited blood loss during the procedure. Use of only local anes-

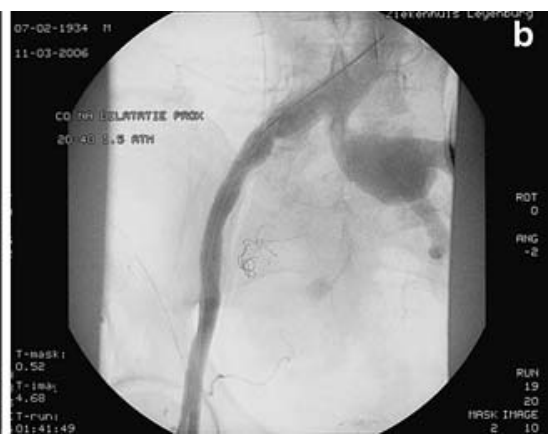

medium is still visible outside the arteries indicating an endoleak (black arrows). b After the second dilatation, no endoleak or enhancement of the aneurysm is seen

thesia is an advantage, especially for high-risk patients. In addition obesity and other factors that hamper anatomic dissection are far less important than in open surgery. During the last decade several reports on endovascular treatment of the IIA have appeared, almost all in an elective setting $[3,4]$. There is only one report of a ruptured IIA being successfully treated endovascularly [5].

In the case of a ruptured aneurysm, whether originating from the aorta or an iliac artery, the primary goal that must be achieved is to obtain a stable hemodynamic situation. In aortic aneurysms EVAR has proved to be a fast and reliable alternative to open surgery [6]. In IIA aneurysms an endovascular procedure can also be a fast method to obtain primary hemostasis. Due to the anatomic location the IIA is not directly accessible for stent-graft placement and stentgrafts as used in the aortic procedures can not be used. The endovascular treatment has to consist of two parts: first the branching vessels of the IIA have to be embolized using coils, and thereafter a covered stent has to be placed in the CIA and EIA, covering the ostium of the IIA. A stent-graft alone would allow inflow of blood into the aneurysm via the branching arteries and collateral arteries from the contralateral side. The combination of preventing backflow via collaterals and preventing inflow from the CIA ensures hemostasis.

In our case we obtained a hemodynamically stable situation within half an hour after starting the procedure, first temporarily by placing the coils and an angioplasty balloon, and later definitively by placing a stent-graft in the CIA and EIA across the ostium of the IIA. A problem in these situations, as illustrated in our case, can be the absence of a suitable stent-graft of the right size and length. It is virtually impossible to have all different sizes of stentgrafts in stock. Placing an angioplasty balloon in a position that seals off the ruptured aneurysm can provide the time to obtain the appropriate stent-graft. The appropriate stentgraft can thus be inserted and deployed later, followed by surgical sheath removal under local anesthesia. 


\section{Conclusion}

In an emergency setting in patients with a ruptured aneurysm, immediate treatment is warranted. The aneurysm has to be excluded from the systemic circulation as soon as possible. Emergency endovascular treatment of a ruptured IIA aneurysm is a promising method, especially since open surgical repair can be a rather difficult procedure. It may be possible to obtain hemostasis in a short period of time with a minimally invasive procedure under local anesthesia. Temporary hemostasis can be obtained by embolization of side-branches of the IIA combined with the placement of an angioplasty balloon in the CIA and EIA crossing the ostium of the IIA. Definitive treatment of the IIA aneurysm with a covered stent-graft can be performed later. In elective patients endovascular treatment of IIA aneurysms has shown promising results in the literature. Our single experience shows that the same endovascular method may be applied in the emergency setting.

\section{References}

1. Dix FP, Titi M, Al-Khaffaf H (2005) The isolated internal iliac artery aneurysm: A review. Eur J Vasc Endovasc Surg 30:119-129

2. Parry DJ, Kessel D, Scott DJ (2001) Simplifying the internal iliac artery aneurysm. Ann R Coll Surg Engl 83:302-308

3. Melki JP, Fichelle JM, Cormier F, Marzelle J, Cormier JM (2001) Embolization of hypogastric artery aneurysm: 17 cases. Ann Vasc Surg 15:312-320

4. Fahrni M, Lachat MM, Wildermuth S, Pfammatter T (2003) Endovascular therapeutic options for isolated iliac aneurysms with a working classification. Cardiovasc Intervent Radiol 26:443-447

5. Ricci MA, Najarian K, Healey CT (2002) Successful endovascular treatment of a ruptured internal iliac aneurysm. J Vasc Surg 35:1274-1276

6. May J, White GH, Waugh R, Stephen MS, Chaufourt X, Yut W, Harris JP (1999) Endovascular treatment of abdominal aortic aneurysms. Cardiovasc Surg 7:484-490 